\title{
Low-dose X-rays leave scars on human hematopoietic stem and progenitor cells: the role of reactive oxygen species
}

\author{
Masayuki Yamashita ${ }^{1}$ and Toshio Suda ${ }^{2,3}$ \\ 'Division of Stem Cell and Molecular Medicine, Center for Stem Cell Biology and Regenerative Medicine, The Institute of Medical Science, \\ The University of Tokyo, Tokyo, Japan; International Research Center for Medical Sciences, Kumamoto University, Kumamoto, Japan and \\ ${ }^{3}$ Cancer Science Institute, National University of Singapore, Singapore
}

In the article by Yamashita M and Suda T entitled "Low-dose X-rays leave scars on human hematopoietic stem and progenitor cells: the role of reactive oxygen species" published in Haematologica 2020;105(8):1986-1988:

\section{$X$-ray has been replaced with ionizing radiation as highlighted in the text below.}

\section{Low-dose ionizing radiations leave scars on human hematopoietic stem and progenitor cells: the role of reactive oxygen species}

\author{
Masayuki Yamashita ${ }^{1}$ and Toshio Suda ${ }^{2,3}$ \\ 'Division of Stem Cell and Molecular Medicine, Center for Stem Cell Biology and Regenerative Medicine, The Institute of Medical Science, \\ The University of Tokyo, Tokyo, Japan; 'International Research Center for Medical Sciences, Kumamoto University, Kumamoto, Japan and \\ ${ }^{3}$ Cancer Science Institute, National University of Singapore, Singapore
}

A fter Röntgen's discovery in 1895, an X-ray became a game changer in medicine. ${ }^{1}$ It was discovered as an invisible ray of light that passes through many objects, including human bodies, and visualizes the internal organs and structures as silhouettes. As now seen in medical radiography, such as chest X-rays and computed tomography (CT) scans, ionizing radiation (IR) has enabled investigation of deep tissues in humans that had been otherwise impossible without surgical intervention, contributing to the early detection and treatment of many diseases. However, as is often the case with new medicine, were shown to have a biohazard effect. ${ }^{2}$ They are identified as a type of IR: a stream of high energy photons that are strong enough to ionize atoms and disrupt molecular bonds in biomolecules, including DNA. As DNA encodes an essential blueprint of a cell, the DNA-damaging property of IR can be toxic. This effect, although used for killing cancer cells in radiotherapy, has raised concerns about the effect of IR on normal tissues and whether the benefits exceed the risks.

Modern medicine relies heavily on radiography to assess human health. The annual doses of IR people receive are increasing. A recent study estimated that around $2 \%$ or $4,000,000$ of the non-elderly adults in the US receive 20 milligray (mGy) or more per year due to medical requirements. ${ }^{3}$ Historically, risks associated with low-dose IR are considered to be almost negligible as it does not cause any acute toxicity, nor does it increase the risk of carcinogenesis, based on empirical linear fits of existing human data determined at high doses, such as those of Japanese atomic bomb survivors. ${ }^{4}$ Indeed, low-dose IR rarely induces DNA double strand breaks (DSB), which often cause mutations and are considered to be the most relevant lesion for the deleterious effects of IR. ${ }^{5}$ However, even though low-dose IR rarely cause DSB, they are reportedly less easy to repair than those induced by high-dose IR . ${ }^{6}$ Importantly, recent evidence suggests that cumulative doses of $50 \mathrm{mGy}$ IR (doses equivalent to 5-10 brain CT scans when given in childhood) have long- term detrimental effects on human health, including a more than 3-fold increase in the risks of acute lymphoblastic leukemia and myelodysplastic syndrome. ' Furthermore, mouse studies demonstrate that low-dose IR affect function of long-lived tissue-specific stem cells, including hematopoietic stem cells (HSC). ${ }^{89}$ Thus, understanding the persistent effect of low-dose IR on human tissue-specific stem cells is of particular importance in precisely evaluating the risks posed by radiography on public health.

In this issue of Haematologica, Henry et al. compared the effects of low and high doses of IR on hematopoietic stem and progenitor cells (HSPC) obtained from human umbilical cord blood (CB) (Figure 1). ${ }^{10}$ HSPC sustain themselves via self-renewing ability, and give rise to all of the blood lineage cells, such as innate and acquired immune cells, erythrocytes and platelets, through multi-lineage differentiation. They found that a single dose of $20 \mathrm{mGy}$ IR is sufficient to impair the self-renewing capacity of CB HSPC. Intriguingly, this effect is independent of canonical DNA damage response (DDR), as a $20 \mathrm{mGy}$ dose fails to induce DSB markers $\gamma$ H2AX and 53BP1 foci, or DDR hallmarks phospho-ATM and -p53, all of which are induced by a 2.5 Gy dose. Instead, the authors demonstrate that it is mediated by reactive oxygen species (ROS), a highly reactive oxygen byproduct mainly generated via the cell respiratory process of oxidative phosphorylation (OXPHOS) in mitochondria, and p38/MAPK14, a key enzyme that, upon elevation of ROS, sends a signal to HSPC to inhibit their self-renewing potential. ${ }^{11}$ Thus, the results of Henry et al. indicate that low-dose IR impair human CB HSPC function through ROS and p38/MAPK14, but not via canonical DDR via ATM or p53.

The high sensitivity of HSC to elevated levels of ROS is well established, first in ATM deficiency and later in the contexts of other stress conditions. ${ }^{11-13}$ Similarly, p38/MAPK14 activation in response to ROS elevation is identified as a common downstream pathway responsible for impairment of self-renewal in HSC. ${ }^{11,12}$ In contrast, what 

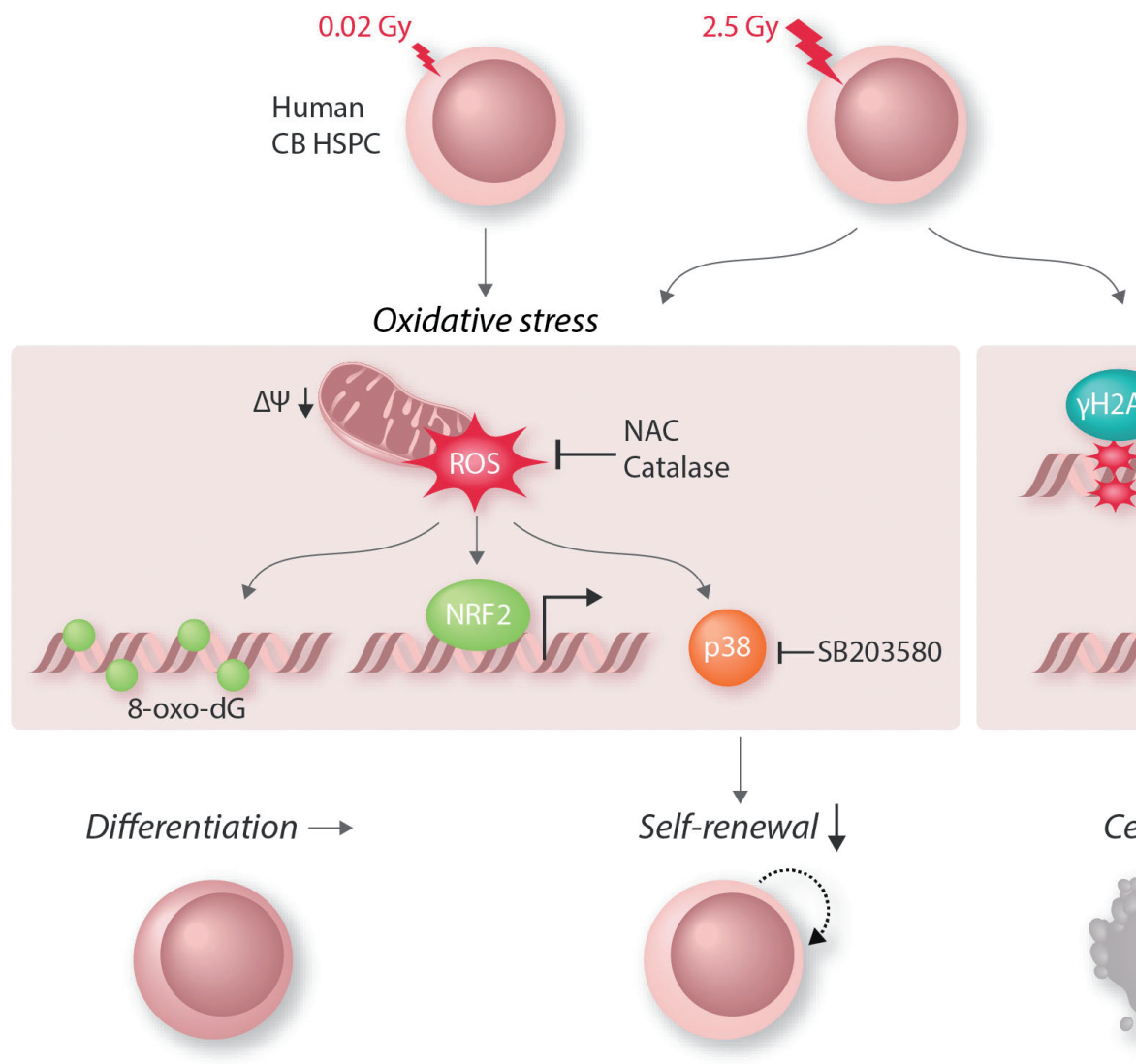

Oxidative stress
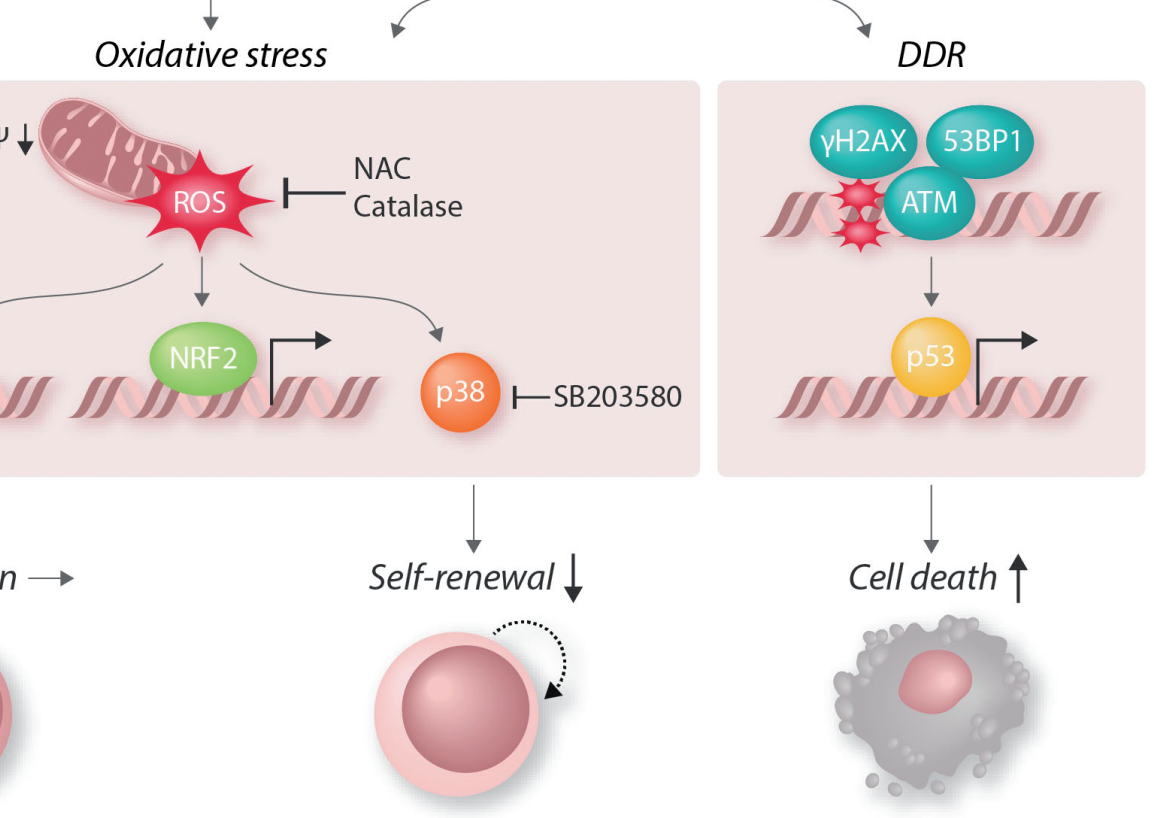

Figure 1. Response of human cord blood (CB) hematopoietic stem and progenitor cells (HSPC) to low- and high-dose ionizing radiation (IR) irradiation demonstrated by Henri et al..$^{10}$ A low dose of 0.02 Gy (20 mGy) IR induces reactive oxygen species (ROS) elevation coupled with decrease in mitochondrial membrane potential $(\Delta \Psi)$, which leads to increase in oxidative stress represented by formation of 8-oxo-deoxyguanosine (8-oxo-dG) in DNA, nuclear expression of NRF2, and activation of p38/MAPK14. The p38/MAPK14 activation mediates a decline in self-renewing capacity of HSPC without affecting their differentiating potential. The low-dose IR do not induce $\gamma-\mathrm{H} 2 \mathrm{AX}$ and 53BP1 foci that represents nuclear DNA double strand breaks (DSB), or canonical DNA damage response via phosphorylation of ATM and p53. In contrast, a high dose of 2.5 Gy IR irradiation causes both ROS elevation and nuclear DSB. As a result, ROS inhibition either by N-acetylcysteine (NAC) or catalase, or p38 inhibition by SB203580, can reverse the detrimental effect by low dose, but not high dose, of IR on self-renewal capacity of HSPC.

is often unclear is the upstream mediator that causes ROS elevation. In the context of low-dose IR, mouse studies have uncovered the hypersensitivity of HSC and esophageal stem cells to low-dose IR that is mediated by ROS elevation, although the molecular link between low-dose IR and elevated ROS has not yet been investigated. ${ }^{8,9}$ It is estimated that approximately $90 \%$ of ROS can be generated during OXPHOS in mitochondria, ${ }^{14}$ mainly through functions of complexes I and III. ${ }^{15}$ Interestingly, the results shown by Henry et al. indicate that ROS elevation in human CB HSPC upon exposure to 20 mGy IR is closely associated with loss of mitochondrial membrane potential, which reflects a decrease in proton gradient across the cristae and often correlates with mitochondrial dysfunction. ${ }^{10}$ Apart from nucleus, mitochondria are the only organelle in mammalian cells that contain DNA, which can also be damaged by low-dose IR. ${ }^{16}$ Mitochondrial DNA (mtDNA) encodes proteins that consist of complexes I and ATP synthase, both of which are essential for proper electron transport and OXPHOS. Of note, these components are located in the so-called "common deletion" region of mtDNA that is commonly deleted upon exposure to low-dose IR. mtDNA is not protected by histones, and is thus potentially more susceptible to IR-induced damage compared to nuclear DNA. Moreover, mtDNA is located in matrix inside inner membranes where ROS is generated, and is thus more greatly affected by IR-induced oxidative stress than nuclear DNA. Damage in mtDNA is not so simple as that in nuclear DNA, as a cell can contain more than 1,000 copies of mtDNA. Furthermore, numbers of mitochondria are dynamically changed by fusion and fission, which play critical roles in maintaining functional mitochondria via inter-mitochondrial complementation and quality control. ${ }^{17}$ In addition, damaged mitochondria can be removed by autophagy, which contributes to maintenance of self-renewal capacity of HSC. ${ }^{18,19}$ Henry et al. show that mitochondrial mass in HSPC does not seem to change after irradiation of $20 \mathrm{mGy}$ IR. ${ }^{10}$ Although this observation should be validated by other methods, ${ }^{20}$ it supports the idea that changes in mitochondrial mass are unlikely to be the cause of ROS elevation. Rather, it is tempting to speculate that damage in mtDNA induced by low-dose IR causes persistent changes in mitochondrial function that lead to initial elevation of ROS and longterm impairment of HSC function. This would be consistent with the results reported by Rodrigues-Moreira et al., which demonstrate that low-dose IR cause biphasic elevations of ROS and the second wave of ROS elevation causes persistent reduction in self-renewing capacity of mouse bone marrow HSC. ${ }^{9}$ Mitochondrial dysfunction, but not constant elevation of ROS, is implicated in age- 
associated decline in HSC function. ${ }^{18}$ Since involvement of mtDNA remains unknown, investigating whether aged HSC have mtDNA damage would be of particular interest. Collectively, identifying molecular 'scars' left by low-dose IR on HSC would help provide a precise evaluation of the long-term detrimental effects by medical radiographic examination and also find common mechanisms that underlie hematopoietic aging and disease.

\section{References}

1. Röntgen WC. On a New Kind of Rays. Science. 1896;3(59):227-231

2. Daniel J. The X-Rays. Science. 1896;3(67):562-563.

3. Fazel R, Krumholz HM, Wang Y, et al. Exposure to low-dose ionizing radiation from medical imaging procedures. $N$ Engl J Med. 2009;361(9):849-857.

4. Preston DL, Kusumi S, Tomonaga $M$, et al. Cancer incidence in atomic bomb survivors. Part III. Leukemia, lymphoma and multiple myeloma, 1950-1987. Radiat Res. 1994;137(2 Suppl):S68-S97.

5. Hoeijmakers JH. Genome maintenance mechanisms for preventing cancer. Nature. 2001;411(6835):366-374.

6. Rothkamm K, Lobrich M. Evidence for a lack of DNA double-strand break repair in human cells exposed to very low $\mathrm{x}$-ray doses. Proc Natl Acad Sci U S A. 2003;100(9):5057-5062.

7. Pearce MS, Salotti JA, Little MP, et al. Radiation exposure from CT scans in childhood and subsequent risk of leukaemia and brain tumours: a retrospective cohort study. Lancet. 2012;380(9840):499505 .

8. Fernandez-Antoran D, Piedrafita G, Murai K, et al. Outcompeting p53-mutant cells in the normal esophagus by redox manipulation. Cell Stem Cell. 2019;25(3):329-341.e6.

9. Rodrigues-Moreira S, Moreno SG, Ghinatti G, et al. Low-dose irradiation promotes persistent oxidative stress and decreases self-renewal in hematopoietic stem cells. Cell Rep. 2017;20(13):3199-3211.

10. Henry E, Souissi-Sahraoui I, Deynoux M, et al. Human hematopoietic stem/progenitor cells display ROS-dependent long-term hematopoietic defects after exposure to low dose of ionizing radiations. Haematologica. 2020;105(8):2044-2055.

11. Ito K, Hirao A, Arai F, et al. Reactive oxygen species act through p38 MAPK to limit the lifespan of hematopoietic stem cells. Nat Med. 2006;12(4):446-451.

12. Miyamoto K, Araki KY, Naka K, et al. Foxo3a is essential for maintenance of the hematopoietic stem cell pool. Cell Stem Cell. 2007;1(1):101-112.

13. Tothova Z, Kollipara R, Huntly BJ, et al. FoxOs are critical mediators of hematopoietic stem cell resistance to physiologic oxidative stress. Cell. 2007;128(2):325-339.

14. Balaban RS, Nemoto S, Finkel T. Mitochondria, oxidants, and aging. Cell. 2005;120(4):483-495.

15. Nissanka N, Moraes CT. Mitochondrial DNA damage and reactive oxygen species in neurodegenerative disease. FEBS Lett. 2018;592(5):728-742

16. Kawamura K, Qi F, Kobayashi J. Potential relationship between the biological effects of low-dose irradiation and mitochondrial ROS production. J Radiat Res. 2018;59(Suppl 2):ii91-ii97.

17. Youle RJ, van der Bliek AM. Mitochondrial fission, fusion, and stress. Science. 2012;337(6098):1062-1065.

18. Ho TT, Warr MR, Adelman ER, et al. Autophagy maintains the metabolism and function of young and old stem cells. Nature. 2017:543 (7644):205-210

19. Ito K, Turcotte R, Cui J, et al. Self-renewal of a purified Tie2+ hematopoietic stem cell population relies on mitochondrial clearance. Science. 2016;354(6316):1156-1160.

20. de Almeida MJ, Luchsinger LL, Corrigan DJ, Williams LJ, Snoeck HW. Dye-Independent Methods Reveal Elevated Mitochondrial Mass in Hematopoietic Stem Cells. Cell Stem Cell. 2017;21(6):725 729.e4 